\title{
A NOTE ON THE DIRICHLET PROBLEM FOR THE STOKES SYSTEM IN LIPSCHITZ DOMAINS
}

\author{
ZHONGWEI SHEN
}

(Communicated by Barbara Lee Keyfitz)

\begin{abstract}
We study the $L^{p}$ Dirichlet problem for the Stokes system in Lipschitz domains. Optimal estimates are obtained when the dimension $n=3$. In the case of $n \geq 4$, we establish a weak estimate of solutions for certain range of $p$.
\end{abstract}

\section{INTRODUCTION}

Let $\Omega$ be a bounded Lipschitz domain in $\mathbf{R}^{n}, n \geq 3$, with connected boundary. Let $N=N(Q)$ denote the outward unit normal to $\partial \Omega$. Consider the Dirichlet problem for the Stokes system:

$$
\begin{cases}\Delta \vec{u}-\nabla p=\overrightarrow{0} & \text { in } \Omega, \\ \operatorname{div} \vec{u}=0 & \text { in } \Omega, \\ \vec{u}=\vec{g} \in L^{q}(\partial \Omega) & \text { on } \partial \Omega .\end{cases}
$$

In [FKV, Theorem 3.9, p. 789], it is shown that there exists $\epsilon=\epsilon(\Omega)>0$, such that, if $\vec{g} \in L^{q}(\partial \Omega), 2-\epsilon<q<2+\epsilon$, and verifies the necessary condition $\int_{\partial \Omega} \vec{g} \cdot N=0$, then there exists a unique $\vec{u}$ and a unique $p$ (modulo constants) satisfying $(0.1)$ and $(\vec{u})^{*} \in L^{q}(\partial \Omega)$, where $(\vec{u})^{*}$ denotes the nontangential maximal function of $\vec{u}$. In this note, we obtain the optimal $L^{q}$-estimates for $2 \leq q \leq \infty$ when the dimension $n=3$ and a weak estimate of solutions for certain range of $q$ in the case of $n \geq 4$.

Let

$$
L_{N}^{q}(\partial \Omega)=\left\{\vec{g} \in L^{q}(\partial \Omega): \int_{\partial \Omega} \vec{g} \cdot N=0\right\} .
$$

Our main results are as follows:

Theorem 0.2. Let $\Omega$ be a bounded Lipschitz domain in $\mathbf{R}^{3}$, with connected boundary. Given $\vec{g} \in L_{N}^{\infty}(\partial \Omega)$, there exists a unique $\vec{u}$ and a $p$ (unique up to a constant) satisfying $(0.1)$ and $\vec{u} \in L^{\infty}(\Omega)$. In fact,

$$
\|\vec{u}\|_{L^{\infty}(\Omega)} \leq C\|\vec{g}\|_{L^{\infty}(\partial \Omega)} .
$$

Received by the editors February 1, 1993 and, in revised form, April 27, 1993 and June 11, 1993.

1991 Mathematics Subject Classification. Primary 35Q30.

Supported in part by the National Science Foundation. 
Moreover, if $\vec{g} \in \Lambda^{\alpha}(\partial \Omega), 0<\alpha<\alpha_{0}=\alpha_{0}(\Omega)$, then $\vec{u} \in \Lambda^{\alpha}(\Omega)$ and

$$
\|\vec{u}\|_{\Lambda^{\alpha}(\Omega)}+\sup _{X \in \Omega} \delta(X)^{1-\alpha}|\nabla \vec{u}(X)| \leq C\|\vec{g}\|_{\Lambda^{\alpha}(\partial \Omega)} .
$$

In Theorem 0.2, $\Lambda^{\alpha}$ denotes the space of Hölder continuous functions of order $\alpha$ and $\delta(X)=\operatorname{dist}(X, \partial \Omega)$.

Theorem 0.3. Let $\Omega$ be a bounded Lipschitz domain in $\mathbf{R}^{3}$, with connected boundary. Given $\vec{g} \in L_{N}^{q}(\partial \Omega), 2 \leq q<\infty$, there exists a unique $\vec{u}$ and $a$ unique (up to a constant) $p$ satisfying $(0.1)$ and $(\vec{u})^{*} \in L^{q}(\partial \Omega)$. Moreover, we have

$$
\left\|(\vec{u})^{*}\right\|_{L^{q}(\partial \Omega)} \leq C\|\vec{g}\|_{L^{q}(\partial \Omega)} .
$$

Theorem 0.3 follows easily from the $L^{\infty}$-estimates in Theorem 0.2 and the $L^{2}$-estimate in [FKV] by interpolation. It is known that the nontangential estimate in Theorem 0.3 implies the Sobolev-Besov estimate:

$$
\|\vec{u}\|_{L_{1 / q}^{q}(\Omega)} \leq C\|\vec{g}\|_{L^{q}(\partial \Omega)}
$$

for $2 \leq q<\infty($ see $[\mathrm{F}, \mathrm{JK}])$.

Theorem 0.4. Let $\Omega$ be a bounded Lipschitz domain in $\mathbf{R}^{n}, n \geq 4$, with connected boundary and $2 \leq q \leq 2(n-1)(n-2) /(n(n-3))$. Then, for any $\vec{g} \in L_{N}^{q}(\partial \Omega)$, the unique $L^{2}$-solution to (0.1) given in [FKV, Theorem 3.9] satisfies

where $q_{1}=n q /(n-1)$.

$$
\|\vec{u}\|_{L^{q_{1}(\Omega)}} \leq C\|\vec{g}\|_{L^{q}(\partial \Omega)}
$$

As a consequence of Theorem 0.2 , we state without a proof the following theorem for the homogeneous Dirichlet problem:

$$
\begin{cases}\Delta \vec{u}-\nabla p=\vec{f} & \text { in } \Omega, \\ \operatorname{div} \vec{u}=0 & \text { in } \Omega, \\ \vec{u}=\overrightarrow{0} & \text { on } \partial \Omega .\end{cases}
$$

Theorem 0.6. Let $\Omega$ be a bounded Lipschitz domain in $\mathbf{R}^{3}$ and $3 / 2 \leq q \leq 3$. Given any $\vec{f} \in W_{-1}^{q}(\Omega)$, there exists a unique $\vec{u}$ and a unique (up to a constant) $p$ satisfying (0.5) and $\vec{u} \in W_{1}^{q}(\Omega)$. Moreover,

$$
\|\vec{u}\|_{W_{1}^{q}(\Omega)}+\|p\|_{L^{q}(\Omega)} \leq C\|\vec{f}\|_{W_{-1}^{q}(\Omega)} .
$$

The proof of Theorem 0.6 may be carried out using Theorem 0.2 , the $L^{2}$ estimates in [FKV], and the argument of D. Jerison and C. Kenig in [JK] where the analogous results for the Poisson's equation are proved.

For the Lipschitz domain in $\mathbf{R}^{3}$, the solvability of the boundary value problems with data in $L^{q}, 2 \leq q \leq \infty$, has been established for the system of elastostatics in [DK2] and for the biharmonic equation in [PV]. Our approach to the Stokes system follows the ideas of B. Dahlberg and C. Kenig and J. Pipher and G. Verchota. The key step is to show that the Green's function $G(X, Y)$ has certain decay when $|X-Y|$ is large in comparison with $\delta(X)$. To do this, we use the Rellich identity, Caccioppoli's inequality, and the fact that the Dirichlet problem is solvable for data in $L^{q}(\partial \Omega)$ for some $q<2$. In the case 
of $n=3$, the estimates on Green's function yields the desired $L^{\infty}$-estimates of solutions. But, when $n \geq 4$, it only gives some weak estimates.

We remark that in [PV], it is shown that, when the dimension $n \geq 4$, the $L^{q}$ Dirichlet problem for the biharmonic equation in general is not solvable in Lipschitz domains for $q>2$ large enough $(n=4, q>6 ; n \geq 5, q>4)$. The counterexample they found is given by $\Omega=O \times \mathbf{R}_{+}$where $O$ is an open subset of the unit sphere in $\mathbf{R}^{n}, n \geq 4$. It is noted in [DK2] that such domains fail to produce counter-examples for the system of elastostatics. In fact, the argument in [MP] implies that the $L^{\infty}$-estimate does hold for the Stokes system and the system of elastostatics in this type of Lipschitz domains.

The outline of this paper is as follows. In section 1 we state and prove the main Lemma (Lemma 1.7) of this paper concerning the estimates on Green's functions. Theorems 0.2 and 0.4 are proved in section 2 .

Finally, the author would like to thank Carlos Kenig for many helpful conversations.

\section{Main Lemma}

We start by recalling the main results in [FKV].

Theorem 1.1. Let $\Omega$ be a bounded Lipschitz domain with connected boundary in $\mathbf{R}^{n}, n \geq 3$. If $\vec{g} \in L_{N}^{2}(\partial \Omega)$, there exists a unique $\vec{u}$ and a unique (up to constants) $p$ satisfying $(0.1)$ and $(\vec{u})^{*} \in L^{2}(\partial \Omega)$. In fact, the solution will satisfy

$$
\left\|(\vec{u})^{*}\right\|_{L^{2}(\partial \Omega)} \leq C\|\vec{g}\|_{L^{2}(\partial \Omega)}
$$

where $C$ depends only on the Lipschitz character of $\partial \Omega$. If, in addition, $\vec{g} \in$ $W_{1}^{2}(\partial \Omega) \cap L_{N}^{2}(\partial \Omega)$, i.e., $\vec{g}$ has first-order derivatives in $L^{2}(\partial \Omega)$, then

$$
\left\|(\vec{u})^{*}\right\|_{L^{2}(\partial \Omega)}+\left\|(\nabla \vec{u})^{*}\right\|_{L^{2}(\partial \Omega)}+\left\|(p)^{*}\right\|_{L^{2}(\partial \Omega)} \leq C\|\vec{g}\|_{W_{1}^{2}(\partial \Omega)} .
$$

Moreover, there exists $\epsilon_{0}=\epsilon_{0}(\Omega)>0$ (which depends only on the Lipschitz character of $\partial \Omega)$, so that the above results extend to $L^{p}(\partial \Omega), W_{1}^{p}(\partial \Omega)$ for $2-\epsilon_{0} \leq p \leq 2+\epsilon_{0}$.

Unless otherwise indicated, we will assume that $\Omega$ is a bounded Lipschitz domain with connected boundary in $\mathbf{R}^{n}, n \geq 3$, throughout this section. We will use $C$ and $c$ to denote constants which depend only on $n, q$, and the Lipschitz character of $\partial \Omega$.

Let $\Gamma(X)=\left(\Gamma_{i j}(X)\right)_{1 \leq i, j \leq n}$ be the matrix of fundamental solutions and $q(X)=\left(q^{j}(X)\right)_{1 \leq j \leq n}$ be the corresponding pressure vector where

$$
\Gamma_{i j}(X)=\frac{1}{2 \omega_{n}}\left(\frac{1}{(n-2)} \frac{\delta_{i j}}{|X|^{n-2}}+\frac{X_{i} X_{j}}{|X|^{n}}\right), \quad q^{j}(X)=\frac{1}{\omega_{n}} \frac{X_{j}}{|X|^{n}} .
$$

Using Theorem 1.1, we can construct the matrix Green's function $G(X, Y)=$ $\left(G_{i j}(X, Y)\right)_{1 \leq i, j \leq n}$ and the corresponding pressure vector $\left(P_{j}^{X}(Y)\right)_{1 \leq j \leq n}$ where

$$
G(X, Y)=\Gamma(X-Y)-v^{X}(Y), \quad P^{X}(Y)=q(X-Y)-p^{X}(Y)
$$


and, for $X \in \Omega,\left(v^{X}, p^{X}\right)$ is the matrix-valued solution to the Dirichlet problem $(0.1)$ with boundary data

$$
v^{X}(Q)=\Gamma(X-Q) \quad \text { on } \partial \Omega \text {. }
$$

Lemma 1.4. Let $X \in \Omega, P \in \partial \Omega$, and $r=|X-P| \leq 2 \operatorname{dist}(X, \partial \Omega)$. Then

$$
\int_{\partial \Omega \backslash \Delta(P, 10 r)}\left|(G(X, \cdot))^{*}(Q)\right|^{q} d Q \leq C r^{(n-1)-(n-2) q}
$$

if $2-\epsilon_{0} \leq q \leq 2+\epsilon_{0}$, where $\epsilon_{0}$ is the same as in Theorem 1.1 and $\Delta(P, r)=$ $B(P, r) \cap \partial \Omega=\{Q \in \partial \Omega:|Q-P|<r\}$.

Proof. We apply Theorem 1.1 on the domain $\Omega \backslash B(P, 4 r)$ to obtain

$$
\begin{aligned}
& \int_{\partial \Omega \backslash \Delta(P, 10 r)}\left|(G(X, \cdot))^{*}(Q)\right|^{q} d Q \\
& \quad \leq C \int_{\Omega \cap \partial B(P, 4 r)}|G(X, Q)|^{q} d Q \\
& \quad \leq C \int_{\Omega \cap \partial B(P, 4 r)}|\Gamma(X-Q)|^{q} d Q+C \int_{\Omega \cap \partial B(P, 4 r)}\left|v^{X}(Q)\right|^{q} d Q
\end{aligned}
$$

where we used (1.3) and the fact that $G(X, \cdot)=0$ on $\partial \Omega$. Clearly, by (1.2),

$$
\int_{\Omega \cap \partial B(P, 4 r)}|\Gamma(X, Q)|^{q} d Q \leq C r^{(n-1)-(n-2) q} \text {. }
$$

Also, note that

$$
\begin{aligned}
& \int_{\Omega \cap \partial B(P, 4 r)}\left|v^{X}(Q)\right|^{q} d Q \\
& \leq C r^{(n-1)\left(1-\frac{q}{2+\epsilon_{0}}\right)}\left(\int_{\Omega \cap \partial B(P, 4 r)}\left|v^{X}(Q)\right|^{2+\epsilon_{0}} d Q\right)^{q /\left(2+\epsilon_{0}\right)} \\
& \leq C r^{(n-1)\left(1-\frac{q}{2+\epsilon_{0}}\right)}\left(\int_{\partial \Omega}\left|\left(v^{X}\right)^{*}(Q)\right|^{2+\epsilon_{0}} d Q\right)^{q /\left(2+\epsilon_{0}\right)} \\
& \leq C r^{(n-1)\left(1-\frac{q}{2+\epsilon_{0}}\right)}\left(\int_{\partial \Omega}|\Gamma(X-Q)|^{2+\epsilon_{0}} d Q\right)^{q /\left(2+\epsilon_{0}\right)} \\
& \leq C r^{(n-1)\left(1-\frac{q}{2+\epsilon_{0}}\right)}\left(\int_{c r}^{\infty} \frac{t^{n-2}}{t^{(n-2)\left(2+\epsilon_{0}\right)}} d t\right)^{q /\left(2+\epsilon_{0}\right)} \\
& \leq C r^{(n-1)-(n-2) q} .
\end{aligned}
$$

The lemma then follows.

The following Caccioppoli's inequality is contained in [GM, p. 203, Theorem 2.2]. Although it is not stated for Lipschitz domains there, the proof can be carried out in the same manner with minor changes.

Lemma 1.5 (Caccioppoli Inequality). Let $X_{0} \in \bar{\Omega}, R>0$ be small, and $D\left(X_{0}, R\right)=B\left(X_{0}, R\right) \cap \Omega$. Assume $(\vec{u}, p)$ is a solution of the Stokes system in $D\left(X_{0}, 3 R\right)$ and $\vec{u}=\overrightarrow{0}$ on $B\left(X_{0}, 3 R\right) \cap \partial \Omega$. Then

$$
\int_{D\left(X_{0}, R\right)}|\nabla \vec{u}|^{2} d X \leq \frac{C}{R^{2}} \int_{D\left(X_{0}, 2 R\right)}|\vec{u}|^{2} d X .
$$


Define the conormal derivative

$$
\frac{\partial \vec{u}}{\partial \nu_{Q}}=\frac{\partial \vec{u}}{\partial N(Q)}-p N(Q)
$$

The following is the main lemma of this paper.

Lemma 1.7 (Main Lemma). There exists an $\epsilon_{1}>0$, such that, if $X \in \Omega, P \in$ $\partial \Omega$, and $r=|X-P| \leq 2 \operatorname{dist}(X, \partial \Omega)$, then

$$
\int_{R \leq|Q-P| \leq 2 R}\left|\frac{\partial G}{\partial \nu_{Q}}(X, Q)-P^{X}\left(X_{0}\right) N(Q)\right|^{2} d Q \leq C\left(\frac{r}{R}\right)^{\epsilon_{1}} \frac{1}{R^{2} r^{n-3}}
$$

where $R \geq 30 r$ and $X_{0}$ is some point in $\Omega$ which does not depend on $R$.

Proof. Fix $X_{0} \in \Omega$ such that $X_{0}$ is away from $X$ and $\operatorname{dist}\left(X_{0}, \partial \Omega\right) \geq c>0$. For $\tau \in[1,3]$, let

$$
D(\tau R)=\{Y \in \Omega: R / \tau \leq|Y-P| \leq \tau R\}
$$

It follows from part (i) of Lemma 1.16 in [FKV, p. 779] and a rescaling argument that

$$
\begin{aligned}
& \int_{R \leq|Q-P| \leq 2 R}\left|\frac{\partial G}{\partial \nu_{Q}}(X, Q)-P^{X}\left(X_{0}\right) N(Q)\right|^{2} d Q \\
& \quad \leq C \int_{\partial D(\tau R)}\left|\frac{\partial G}{\partial \nu_{Q}}(X, Q)-P^{X}\left(X_{0}\right) N(Q)\right|^{2} d Q \\
& \quad \leq C \int_{\partial D(\tau R)}\left|\nabla_{\tan } G(X, Q)\right|^{2} d Q+C R^{n-1}\left|P^{X}\left(X_{R}\right)-P^{X}\left(X_{0}\right)\right|^{2}
\end{aligned}
$$

where $\nabla_{\tan }$ denotes the tangential derivative on $\partial \Omega$ and $X_{R}$ is a point in $D(R)$ such that $\operatorname{dist}\left(X_{R}, \partial \Omega\right) \sim R$.

Integrating in $\tau \in[1,3 / 2]$, we have

$$
\begin{aligned}
\int_{R \leq|Q-P| \leq 2 R}\left|\frac{\partial G}{\partial \nu_{Q}}(X, Q)-P^{X}\left(X_{0}\right) N(Q)\right|^{2} d Q \\
\quad \leq \frac{C}{R} \int_{D\left(\frac{3}{2} R\right)}|\nabla G(X, Y)|^{2} d Y+C R^{n-1}\left|P^{X}\left(X_{R}\right)-P^{X}\left(X_{0}\right)\right|^{2} \\
\quad \leq \frac{C}{R^{3}} \int_{D(2 R)}|G(X, Y)|^{2} d Y+C R^{n-1}\left|P^{X}\left(X_{R}\right)-P^{X}\left(X_{0}\right)\right|^{2}
\end{aligned}
$$

where we used the fact that $G(X, \cdot)=0$ on $\partial \Omega$ in the first inequality and Caccioppoli's inequality (Lemma 1.5) in the second inequality.

We first estimate the integral which involves the Green's function. 
It follows from Hölder's inequality and the Sobolev inequality that

$$
\begin{aligned}
\frac{1}{R^{3}} & \int_{D(2 R)}|G(X, Y)|^{2} d Y \\
\leq & C R^{\frac{n-4}{2}-\frac{n}{2-\epsilon_{0}}}\left(\int_{D(2 R)}|G(X, Y)|^{2-\epsilon_{0}} d Y\right)^{1 /\left(2-\epsilon_{0}\right)} \\
& \times\left(\int_{D(2 R)}|G(X, Y)|^{\frac{2 n}{n-2}} d Y\right)^{(n-2) /(2 n)} \\
\leq & C R^{\frac{n-4}{2}-\frac{n}{2-\epsilon_{0}}}\left(\int_{D(2 R)}|G(X, Y)|^{2-\epsilon_{0}} d Y\right)^{1 /\left(2-\epsilon_{0}\right)}\left(\int_{D(2 R)}|\nabla Y G(X, Y)|^{2} d Y\right)^{1 / 2} \\
\leq & C R^{\frac{n-6}{2}-\frac{n}{2-\epsilon_{0}}}\left(\int_{D(2 R)}|G(X, Y)|^{2-\epsilon_{0}} d Y\right)^{1 /\left(2-\epsilon_{0}\right)}\left(\int_{D(3 R)}|G(X, Y)|^{2} d Y\right)^{1 / 2} \\
\leq & C R^{\frac{n-5}{2}-\frac{n-1}{2-\epsilon_{0}}}\left(\int_{\partial \Omega \backslash \Delta(P, 10 r)}\left|(G(X, \cdot))^{*}(Q)\right|^{2-\epsilon_{0}} d Q\right)^{1 /\left(2-\epsilon_{0}\right)} \\
& \times\left(\int_{\partial \Omega \backslash \Delta(P, 10 r)}\left|(G(X, \cdot))^{*}(Q)\right|^{2} d Q\right)^{1 / 2} \\
\leq & C\left(\frac{r}{R}\right)^{\epsilon_{1}} \cdot \frac{1}{R^{2} r^{n-3}}
\end{aligned}
$$

where $\epsilon_{1}=(n-1)\left\{1 /\left(2-\epsilon_{0}\right)-1 / 2\right\}$ and we used Lemma 1.4 in the last inequality.

Finally we need to estimate the term $R^{n-1}\left|P^{X}\left(X_{R}\right)-P^{X}\left(X_{0}\right)\right|^{2}$.

Since $\Omega$ is a Lipschitz domain, there exists a curve $\gamma(t), t \in[0,1]$, such that $\gamma(0)=X_{R}, \gamma(1)=X_{0},\left|\gamma^{\prime}(t)\right| \sim 1$, and $\delta(\gamma(t)) \sim|\gamma(t)-P|$.

Clearly,

$$
\begin{aligned}
\left|P^{X}\left(X_{R}\right)-P^{X}\left(X_{0}\right)\right| & \leq \int_{0}^{1}\left|\frac{d}{d t} P^{X}(\gamma(t))\right| d t \leq C \int_{0}^{1}\left|\left(\nabla P^{X}\right)(\gamma(t))\right| d t \\
& =C \int_{0}^{1}\left|\left(\Delta_{Y} G\right)(X, \gamma(t))\right| d t .
\end{aligned}
$$

By the well-known interior estimates for the solutions of Stokes system, if $|Y-P| \sim \delta(Y)$,

$$
\begin{aligned}
\left|\Delta_{Y} G(X, Y)\right| & \leq \frac{C}{(\delta(Y))^{n+2}} \int_{B\left(Y, \frac{c \delta(Y)}{2}\right)}|G(X, Z)| d Z \\
& \leq \frac{C}{(\delta(Y))^{2}} \cdot \frac{1}{R^{n-1}} \int_{R \leq|Q-P| \leq 2 R}\left|(G(X, \cdot))^{*}(Q)\right| d Q
\end{aligned}
$$

Hence,

$$
\begin{aligned}
\left|P^{X}\left(X_{R}\right)-P^{X}\left(X_{0}\right)\right| & \leq C \int_{0}^{1} \frac{d t}{|\delta(\gamma(t))|^{2}} \cdot \frac{1}{R^{n-1}} \int_{R \leq|Q-P| \leq 2 R}\left|(G(X, \cdot))^{*}(Q)\right| d Q \\
& \leq \frac{C}{R^{n}} \int_{R \leq|P-Q| \leq 2 R}\left|(G(X, \cdot))^{*}(Q)\right| d Q .
\end{aligned}
$$

The desired estimate follows easily from Hölder's inequality and Lemma 1.4. The lemma is then proved. 
Lemma 1.8. Let $\Omega$ be a bounded Lipschitz domain in $\mathbf{R}^{3}$, with connected boundary. Then, there exists an $\alpha_{0}>0$, which depends only on the Lipschitz character of $\partial \Omega$, such that, if $X \in \Omega, P \in \partial \Omega$, and $r=|X-P| \leq 2 \delta(X)$, then

$$
\int_{\partial \Omega}\left|\frac{\partial G}{\partial \nu_{Q}}(X, Q)-P^{X}\left(X_{0}\right) N(Q)\right|(r+|Q-P|)^{\alpha} d Q \leq C r^{\alpha}
$$

where $0 \leq \alpha \leq \alpha_{0}$ and $X_{0}$ is some point in $\Omega$.

Proof. Let $X \in \Omega, P \in \partial \Omega$, and $r=|X-P| \leq 2 \delta(X)$. It follows from Hölder's inequality and Lemma 1.7 that, for $R \geq 30 r$,

$$
\begin{aligned}
\int_{R \leq|Q-P| \leq 2 R} \mid & \frac{\partial G}{\partial \nu_{Q}}(X, Q)-P^{X}\left(X_{0}\right) N(Q) \mid(r+|Q-P|)^{\alpha} d Q \\
& \leq R^{1+\alpha}\left(\int_{R \leq|Q-P| \leq 2 R}\left|\frac{\partial G}{\partial \nu_{Q}}(X, Q)-P^{X}\left(X_{0}\right) N(Q)\right|^{2} d Q\right)^{1 / 2} \\
& \leq C r^{\alpha}\left(\frac{r}{R}\right)^{\epsilon_{1} / 2-\alpha} .
\end{aligned}
$$

Choose $\alpha_{0}$ such that $0<\alpha_{0}<\epsilon_{1} / 2$. It is easy to see that, if $0 \leq \alpha \leq \alpha_{0}$,

$$
\int_{\partial \Omega \backslash \Delta(P, 30 r)}\left|\frac{\partial G}{\partial \nu_{Q}}(X, Q)-P^{X}\left(X_{0}\right) N(Q)\right|(r+|Q-P|)^{\alpha} d Q \leq C r^{\alpha}
$$

To finish the proof, we need to show that

$$
\int_{\Delta(P, 30 r)}\left|\frac{\partial G}{\partial \nu_{Q}}(X, Q)-P^{X}\left(X_{0}\right) N(Q)\right| d Q \leq C .
$$

Recall that $G(X, Y)=\Gamma(X-Y)-v^{X}(Y)$ and $P^{X}(Y)=q(X-Y)-p^{X}(Y)$ (See (1.3)). It is not hard to see that

$$
\int_{\Delta(P, 30 r)}\left|\frac{\partial \Gamma}{\partial \nu_{Q}}(X, Q)-q\left(X-X_{0}\right) N(Q)\right| d Q \leq C .
$$

Also, note that, by the $L^{2}$-estimate for solutions of the Dirichlet problem with data in $W_{1}^{2}(\partial \Omega)$, we have

$$
\begin{aligned}
\int_{\Delta(P, 30 r)}\left|\frac{\partial v^{X}}{\partial \nu_{Q}}(Q)-p^{X}\left(X_{0}\right) N(Q)\right| d Q \\
\quad \leq C r\left(\int_{\Delta(P, 30 r)}\left|\frac{\partial v^{X}}{\partial \nu_{Q}}(Q)-p^{X}\left(X_{0}\right) N(Q)\right|^{2} d Q\right)^{1 / 2} \\
\quad \leq C r\left\|v^{X}\right\|_{W_{1}^{2}(\partial \Omega)} \leq C .
\end{aligned}
$$

The proof is then complete.

\section{The Dirichlet PRoblem}

In this section, we give the proofs for Theorem 0.2 and Theorem 0.4 that we stated in the Introduction.

We begin with

Proof of Theorem 0.2. Uniqueness follows from the uniqueness of the $L^{2}$ solution in [FKV]. We only need to show the existence.

To this end, we first assume $\vec{g} \in L_{N}^{\infty}(\partial \Omega)$.Let $(\vec{u}, p)$ be the $L^{2}$-solution 
with boundary data $\vec{g}$. We have the Poisson integral formula (see [FKV])

$$
\vec{u}(X)=\int_{\partial \Omega}\left(\frac{\partial G}{\partial \nu_{Q}}(X, Q)-P^{X}\left(X_{0}\right) N(Q)\right) \vec{g}(Q) d Q .
$$

By Lemma 1.8,

$$
\int_{\partial \Omega}\left|\frac{\partial G}{\partial \nu_{Q}}(X, Q)-P^{X}\left(X_{0}\right) N(Q)\right| d Q \leq C .
$$

It follows that, for any $X \in \Omega$,

$$
|\vec{u}(X)| \leq C\|\vec{g}\|_{L^{\infty}(\partial \Omega)} .
$$

Next, we consider the case $\vec{g} \in \Lambda^{\alpha}(\partial \Omega)$ where $0<\alpha<\alpha_{0}$.

It is enough to show that

$$
\delta(X)^{1-\alpha}|\nabla \vec{u}(X)| \leq C\|\vec{g}\|_{\Lambda^{\alpha}(\partial \Omega)} \text { for } X \in \Omega .
$$

To do this, we fix $X \in \Omega$ and $P \in \partial \Omega$ such that $|X-P| \leq 2 \delta(X)$. Since $\vec{u}(Y)-\vec{g}(P)$ is a solution with boundary value $\vec{g}-\vec{g}(P)$, we have

$$
\vec{u}(Y)-\vec{g}(P)=\int_{\partial \Omega}\left(\frac{\partial G}{\partial \nu_{Q}}(Y, Q)-P^{Y}\left(Y_{0}\right) N(Q)\right)(\vec{g}(Q)-\vec{g}(P)) d Q .
$$

It then follows from Lemma 1.8 that, if $|Y-X| \leq \delta(X) / 4$,

$$
\begin{aligned}
|\vec{u}(Y)-\vec{g}(P)| & \leq C\|\vec{g}\|_{\Lambda^{\alpha}(\partial \Omega)} \int_{\partial \Omega}\left|\frac{\partial G}{\partial \nu_{Q}}(Y, Q)-P^{Y}\left(Y_{0}\right) N(Q) \| Q-P\right|^{\alpha} d Q \\
& \leq C|X-P|^{\alpha}\|\vec{g}\|_{\Lambda^{\alpha}(\partial \Omega)} .
\end{aligned}
$$

Hence, by the interior estimates,

$$
\begin{aligned}
|\nabla \vec{u}(X)| & \leq \frac{C}{(\delta(X))^{n+1}} \int_{B\left(X, \frac{\delta(X)}{4}\right)}|\vec{u}(Y)-\vec{g}(P)| d Y \\
& \leq \frac{C}{\delta(X)} \cdot|X-P|^{\alpha}\|\vec{g}\|_{\Lambda^{\alpha}(\partial \Omega)} \leq C \delta(X)^{\alpha-1}\|\vec{g}\|_{\Lambda^{\alpha}(\partial \Omega)} .
\end{aligned}
$$

The desired estimate (2.1) then follows. The proof of Theorem 0.2 is complete.

Theorem 0.3 follows easily from the $L^{2}$-estimate in [FKV] and the $L^{\infty}$ estimate in Theorem 0.2 by interpolation. We omit the details.

We will need the following lemma in the proof of Theorem 0.4.

Lemma 2.2. Let $\vec{u} \in C^{\infty}(\Omega)$. Then, for $1<q<\infty$,

$$
\int_{\Omega}|\vec{u}(X)|^{q} \leq C \int_{\Omega}|\delta(X) \nabla \vec{u}(X)|^{q} d X+C \sup _{X \in K}|\vec{u}(X)|^{q}
$$

where $K$ is a compact subset of $\Omega$.

Proof. Fix $P_{0} \in \partial \Omega$ and $r_{0}>0$ small. Without loss of generality, we may assume that there exists a Lipschitz function $\phi: \mathbf{R}^{\mathbf{n}-1} \rightarrow \mathbf{R}$ such that

$$
\begin{aligned}
B\left(P_{0}, 10 r_{0}\right) \cap \Omega & =\left\{\left(X^{\prime}, x_{n}\right): x_{n}>\phi\left(X^{\prime}\right)\right\}, \\
B\left(P_{0}, 10 r_{0}\right) \cap \partial \Omega & =\left\{\left(X^{\prime}, x_{n}\right): x_{n}=\phi\left(X^{\prime}\right)\right\}
\end{aligned}
$$

where $X^{\prime}=\left(x_{1}, x_{2}, \ldots, x_{n-1}\right)$. 
Let $\vec{v}\left(X^{\prime}, x_{n}\right)=\vec{u}\left(X^{\prime}, \phi\left(X^{\prime}\right)+x_{n}\right)$. Then, if $\left|X^{\prime}\right| \leq r_{0}, 0<x_{n}<r_{0}$,

$\left|\vec{v}\left(X^{\prime}, x_{n}\right)\right| \leq \int_{x_{n}}^{r_{0}}\left|\frac{\partial \vec{v}}{\partial t}\left(X^{\prime}, t\right)\right| d t+\left|\vec{v}\left(X^{\prime}, r_{0}\right)\right| \leq \int_{x_{n}}^{r_{0}}\left|\frac{\partial \vec{v}}{\partial t}\left(X^{\prime}, t\right)\right| d t+\sup _{K}|\vec{u}|$

where $K=\left\{X \in \Omega: \delta(X) \geq c_{0}>0\right\}$.

It follows that

$$
\begin{aligned}
\int_{\left|X^{\prime}\right| \leq r_{0}} & \int_{0}^{r_{0}}\left|\vec{v}\left(X^{\prime}, x_{n}\right)\right|^{q} d X^{\prime} d x_{n} \\
& \leq C \int_{\left|X^{\prime}\right| \leq r_{0}} \int_{0}^{r_{0}}\left(\int_{x_{n}}^{r_{0}}\left|\frac{\partial \vec{v}}{\partial t}\left(X^{\prime}, t\right)\right| d t\right)^{q} d x_{n} d X^{\prime}+C \sup _{K}|\vec{u}|^{q} \\
& \leq C \int_{\left|X^{\prime}\right| \leq r_{0}} \int_{0}^{r_{0}}\left(t\left|\frac{\partial \vec{v}}{\partial t}\left(X^{\prime}, t\right)\right|\right)^{q} d t d X^{\prime}+C \sup _{K}|\vec{u}|^{q}
\end{aligned}
$$

where we have used the Hardy inequality in the second inequality (see [S, p. 272]).

Clearly, this implies that

$$
\int_{B\left(P_{0}, r_{0}\right) \cap \Omega}|\vec{u}|^{q} d X \leq C \int_{B\left(P_{0}, 2 r_{0}\right) \cap \Omega}|\delta(X) \nabla \vec{u}|^{q} d X+C \sup _{K}|\vec{u}|^{q} .
$$

The lemma follows by covering the boundary $\partial \Omega$ with a finite collection of coordinate cylinders.

The next theorem concerns the estimates of solutions of $(0.1)$ in terms of the area integral.

Theorem 2.3. Let $\Omega$ be a bounded Lipschitz domain in $\mathbf{R}^{n}, n \geq 3$, with connected boundary. Let $\vec{g} \in L_{N}^{2}(\partial \Omega)$, the unique solution $(\vec{u}, p)$ to the Dirichlet problem (0.1) given in Theorem 1.1 satisfy

$$
\int_{\Omega} \delta(X)|\nabla \vec{u}(X)|^{2} d X \leq C \int_{\partial \Omega}|\vec{g}|^{2} d Q
$$

Theorem 2.3 follows from a general result in [DKJV] which states that the area integral and the nontangential maximal function have comparable $L^{q}(\partial \Omega)$ norm.

We are now in a position to give the proof of Theorem 0.4.

Proof of Theorem 0.4 . Let $\vec{g} \in L^{q_{0}}(\partial \Omega)$ where $q_{0}=2(n-1) /(n-3)$ and $(\vec{u}, p)$ be the $L^{2}$-solution of $(0.1)$ given in [FKV].

We claim that

$$
\delta(X)^{(n-3) / 2}|\vec{u}(X)| \leq C\|\vec{g}\|_{L^{q_{0}}(\partial \Omega)} \quad \text { for } X \in \Omega .
$$

Theorem 0.4 follows from Theorem 2.3 and (2.4) by interpolation. Indeed, by Theorem 2.3,

$$
\left\|\delta(\cdot)^{1 / 2} \nabla \vec{u}(\cdot)\right\|_{L^{2}(\Omega)} \leq C\|\vec{g}\|_{L^{2}(\partial \Omega)} .
$$

On the other hand, it follows from interior estimates and (2.4) that

$$
\left\|\delta(\cdot)^{(n-1) / 2} \nabla \vec{u}(\cdot)\right\|_{L^{\infty}(\Omega)} \leq C\|\vec{g}\|_{L^{q_{0}}(\partial \Omega)}, \quad q_{0}=2(n-1) /(n-3) .
$$


By complex interpolation, estimates (2.5) and (2.6) yield that

$$
\|\delta(\cdot) \nabla \vec{u}(\cdot)\|_{L^{q_{2}}(\Omega)} \leq C\|\vec{g}\|_{L^{q_{1}}(\partial \Omega)} .
$$

where $q_{1}=2(n-1)(n-2) /(n(n-3))$ and $q_{2}=n q_{1} /(n-1)$. Hence, by Lemma 2.2 ,

$$
\|\vec{u}\|_{L^{q_{2}(\Omega)}} \leq C\|\vec{g}\|_{L^{q_{1}(\partial \Omega)}} .
$$

Theorem 0.4 is then proved for the case $q=2(n-1)(n-2) /(n(n-3))$. The case $2 \leq q<2(n-1)(n-2) /(n(n-3))$ follows by interpolation.

Finally, we need to show (2.4).

Fix $X \in \Omega$. Let $P \in \partial \Omega$ such that $r=|X-P|=\delta(X)$. Write

$$
\begin{aligned}
\vec{u}(X) & =\int_{\partial \Omega}\left(\frac{\partial G}{\partial \nu_{Q}}(X, Q)-P^{X}\left(X_{0}\right) N(Q)\right) \vec{g}(Q) d Q \\
& =\int_{|Q-P| \leq 32 r}+\sum_{j=5}^{\infty} \int_{2^{j} r \leq|Q-P| \leq 2^{j+1} r}=I_{0}+\sum_{j=5}^{\infty} I_{j}
\end{aligned}
$$

It follows from Cauchy's inequality and the argument in the proof of Lemma 1.8 that

$$
\begin{aligned}
\left|I_{0}\right| \leq & \int_{|Q-P| \leq 32 r}\left|\frac{\partial G}{\partial \nu_{Q}}(X, Q)-P^{X}\left(X_{0}\right) N(Q) \| \vec{g}(Q)\right| d Q \\
\leq & \left(\int_{|Q-P| \leq 32 r}\left|\frac{\partial G}{\partial \nu_{Q}}(X, Q)-P^{X}\left(X_{0}\right) N(Q)\right|^{2} d Q\right)^{1 / 2} \\
& \times\left(\int_{|Q-P| \leq 32 r}|\vec{g}(Q)|^{2} d Q\right)^{1 / 2} \\
\leq & \frac{C}{r^{(n-1) / 2}}\left(\int_{|Q-P| \leq 32 r}|\vec{g}|^{2} d Q\right)^{1 / 2} \leq \frac{C}{r^{(n-3) / 2}}\|\vec{g}\|_{L^{q_{0}}(\partial \Omega)} .
\end{aligned}
$$

Let $R=2^{j} r$. By Cauchy's inequality and Lemma 1.7,

$$
\begin{aligned}
\left|I_{j}\right| \leq & \left(\int_{R \leq|Q-P| \leq 2 R}\left|\frac{\partial G}{\partial \nu_{Q}}(X, Q)-P^{X}\left(X_{0}\right) N(Q)\right|^{2} d Q\right)^{1 / 2} \\
& \times\left(\int_{R \leq|Q-P| \leq 2 R}|\vec{g}|^{2} d Q\right)^{1 / 2} \\
\leq & C\left(\frac{r}{R}\right)^{\epsilon_{1} / 2} \cdot \frac{1}{R r^{\frac{n-3}{2}}}\left(\int_{R \leq|Q-P| \leq 2 R}|\vec{g}|^{2} d Q\right)^{1 / 2} \\
\leq & C\left(\frac{r}{R}\right)^{\epsilon_{1} / 2} \frac{1}{r^{(n-3) / 2}}\|\vec{g}\|_{L^{q_{0}}(\partial \Omega)} \\
= & C\left(2^{\epsilon_{1} / 2}\right)^{-j} \frac{1}{r^{(n-3) / 2}}\|\vec{g}\|_{L^{q_{0}(\partial \Omega)}} .
\end{aligned}
$$

Hence,

$$
\begin{aligned}
|\vec{u}(X)| & \leq\left|I_{0}\right|+\sum_{j=5}^{\infty}\left|I_{j}\right| \leq \frac{C}{\delta(X)^{(n-3) / 2}}\left\{1+\sum_{j=4}^{\infty}\left(2^{\epsilon_{0} / 2}\right)^{-j}\right\}\|\vec{g}\|_{L^{q_{0}}(\partial \Omega)} \\
& \leq \frac{C}{\delta(X)^{(n-3) / 2}}\|\vec{g}\|_{L^{q_{0}}(\partial \Omega) .}
\end{aligned}
$$

The estimate (2.4) is then proved. The proof of Theorem 0.4 is finished. 


\section{REFERENCES}

[DK1] B. Dahlberg and C. Kenig, Hardy spaces and the Neumann problem in $L^{p}$ for Laplace's equation in Lipschitz domains, Ann. of Math. (2) 125 (1987), 437-465.

[BK2] $\longrightarrow, L^{p}$ estimates for the three-dimension system of elastostatics on Lipschitz domains, Lecture Notes in Pure and Appl. Math., vol. 122, Dekker, New York, 1990.

[DKPV] B. Dahlberg, C. Kenig, J. Pipher, and G. Verchota, Area integral estimates and maximum principles for higher order elliptic equations and systems on Lipschitz domains, preprint.

[DKV] B. Dahlberg, C. Kenig, and G. Verchota, Boundary value problems for the systems of elastostatics in Lipschitz domains, Duke Math. J. 57 (1988), 795-818.

[F] E. Fabes, Layer potential methods for boundary value problems on Lipschitz domains, Lecture Notes in Math., vol. 1344, Springer-Verlag, New York, 1987, pp. 55-80.

[FKV] E. Fabes, C. Kenig, and G. Verchota, The Dirichlet problem for the Stokes system on Lipschitz domains, Duke Math. J. 57 (1988), 769-793.

[GM] M. Giaquinta and G. Modica, Nonlinear systems of the type of the stationary Navier-Stokes system, J. Reine Angew. Math. 330 (1982), 173-214.

[JK] D. Jerison and C. Kenig, The inhomogeneous Dirichlet problem in Lipschitz domains, preprint.

[MP] V. G. Maz'ya and V. A. Plamenvskii, On properties of solutions of three-dimensional problems of elasticity theory and hydrodynamics in domains with isolated singular points, Amer. Math. Soc. Transl. Ser. 2, vol. 123, Amer. Math. Soc., Providence, RI, 1984, pp. 109-123.

[PV] J. Pipher and G. Verchota, The Dirichlet problem in $L^{p}$ for biharmonic functions on Lipschitz domains, Amer. J. Math. 114 (1992), 923-972.

[S] E. Stein, Singular integrals and differentiability properties of functions, Princeton Univ. Press, Princeton, NJ, 1970.

Department of Mathematics, Purdue University, West Lafayette, Indiana 47907

E-mail address: shenz@ math.purdue.edu 